\title{
Effect of Realistic Physical Layer on Energy Efficient Broadcast Protocols for Wireless Ad Hoc Networks
}

\author{
Hui Xu, Manwoo Jeon, Jinsung Cho, Niu Yu and S.Y. Lee ${ }^{1}$ \\ Department of Computer Engineering \\ Kyung Hee University, Korea \\ \{xuhui, imanoos\}@oslab.khu.ac.kr, chojs@khu.ac.kr, \{niuyu, \\ sylee\}@oslab.khu.ac.kr
}

\begin{abstract}
Previous work on energy efficient broadcast protocols for wireless ad hoc networks are based a commonly used physical layer model called "Pathloss model" which assume two nodes can communicate if and only if they exist within their transmission radius. In this paper, we analyze the effect of realistic physical layer on energy efficient broadcast protocols. We employ a more realistic log-normal shadowing model for physical layer and consider two link layer operating models: EER (end-to-end retransmission) and HHR (hop-byhop retransmission). Networks with omni-antennas and directional antennas are dealt with separately. Based on above models, we analyze how to adjust actual transmission radius for transmission nodes and relay nodes to get the trade-off between maximizing probability of delivery and minimizing energy consumption. From our analysis based on shadowing model, we have derived the appropriate transmission range. The results presented in this paper are expected to improve the performance of broadcast protocols under realistic physical layer.
\end{abstract}

\section{Introduction}

Wireless ad hoc networks have emerged recently because of their potential applications in various situations such as battlefield, emergency rescue, and conference environments [1-4]. Ad hoc networks are without a fixed infrastructure; communications take place over a wireless channel, where each node has the ability to communicate with others in the neighborhood, determined by the transmission range. In such network, broadcast is a frequently required operation needed for route discovery, information dissemination, publishing services, data gathering, task distribution, alarming, time synchronization, and other operations. In a broadcasting task, a message is to be sent from one node to all the other ones in the network. Since ad hoc networks are power constrained, the most important design criterion is energy and computation conservation, broadcast is normally completed by multi-hop forwarding. We study position-based efficient broadcast protocols in which location information facilitates efficient broadcasting in terms of selecting a small forward node set and appropriate transmission radiuses while ensuring broadcast coverage. The optimization criterion is minimizing the total transmission power. There exist a lot of energy efficient broadcast protocols and their proposals are as following: first set up broadcast tree,

\footnotetext{
${ }^{1}$ Dr. Sungyoung Lee is the corresponding author.
} 
and then at each transmission the transmission nodes will adjust their transmission radius to the distance between transmission nodes and relay nodes.

However, existing energy efficient broadcast protocols are normally based on a commonly used physical layer model called "Path-loss model" which assume that two nodes can communicate if and only if they exist within their transmission radius. In this paper, we take more realistic models into consideration. For physical layer, we employ a universal and widely-used statistic shadowing model, where nodes can only indefinitely communicate near the edge of the communication range. For link layer, we consider two operating models: EER (end-to-end retransmission without acknowledgement) and HHR (hop-by-hop retransmission with acknowledgement). In addition, energy efficient broadcast protocols in networks with omni-antennas and networks with directional antennas are dealt with separately. Based on realistic physical layer, we apply existing reception probability function and analyze how to choose the actual transmission radius between transmission nodes and relay nodes. We show how the realistic physical layer impact the selection of transmission radius in energy efficient broadcast protocols and present the trade off between maximizing probability of delivery and minimizing energy consumption in the selection of transmission radius. From our analysis, we have derived the appropriate transmission range. The results presented in this paper are expected to improve the performance of broadcast protocols under realistic physical layer.

The remainder of the paper is organized as follows: Section 2 presents related work and offers some critical comments. In Section 3, we introduce our system model, including realistic physical layer and link layer model. In Section 4 we define metrics used in our analysis, i.e. packet reception probability and expected energy consumption. In Section 5 we analyze the effect of realistic physical layer on energy efficient broadcast protocols and derived appropriate transmission radius. In Section 6, we present our conclusions and future work.

\section{Related Work}

In wireless ad hoc networks, the most important design criterion is energy and computation conservation since nodes have limited resources. Except reducing the number of needed emissions, radius adjustment is also a good way to further reduce the energy consumption. Energy efficient broadcast protocols aim to select a small forward node set and appropriate transmission radiuses while ensuring broadcast coverage. Broadcast oriented protocols achieve the objective but considers the broadcast process from a given source node. For example, the well-known centralized algorithm is a greedy heuristics called BIP [5] (Broadcast Incremental Power). It is a variant of the Prim's algorithm that takes advantage of the broadcast nature of wireless transmissions. Basically, a broadcast tree is computed from a source node by adding nodes one at a time. At each step, the less expensive action to add a node is selected, either by increasing the radius of an already transmitting node, or by creating a new emission from a passive one. As a consequence of the "wireless broadcast advantage" property of omni-antennas systems, all nodes whose distance from Node $i$ does not 
exceed $r_{i j}$ will be able to receive the transmission with no further energy expenditure at Node $i$.

The use of directional antennas can permit energy savings and reduce interference by concentrating transmission energy where it is needed. While using directional antenna, the advantage property will be diminished, since only the nodes located within the transmitting node's antenna beam can receive the signal. In Fig. 1 , only $j, l$ can receive the signal, while $k$ cannot receive the signal. Applying the incremental power philosophy to network with directional antennas, the Directional Broadcast Incremental Power (DBIP) algorithm [6] has very good performance in energy saving.

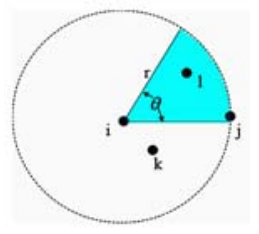

Fig.1. Use of directional antenna

Our work has been inspired by recent research work made in [7-10]. Mineo Takai, et al [7] focused on the effects of physical layer modeling on the performance evaluation of higher layer protocols, and have demonstrated the importance of the physical layer modeling even if the evaluated protocols do not directly interact with the physical layer. The set of relevant factors at the physical layer includes signal reception, path loss, fading, interference and noise computation, and preamble length. I. Stojmenovic, et al [8-10] presented guidelines on how to design routing and broadcasting in ad hoc networks taking physical layer impact into consideration. They apply the log normal shadow fading model to represent a realistic physical layer to derive the approximation for probability $p(d)$ of receiving a packet successfully as a function of distance $d$ between two nodes. They proposed several localized routing schemes for the case when position of destination is known, optimizing expected hop count (for hop by hop acknowledgement), or maximizing the probability of delivery (when no acknowledgements are sent). They considered localized power aware routing schemes under realistic physical layer. Finally, they mentioned broadcasting in ad hoc network with realistic physical layer and propose new concept of dominating sets to be used in broadcasting process.

In this paper, we employ the system model of previous work constructed and analyze the effect of realistic physical layer on energy efficient broadcast protocols.

\section{System Model}

\subsection{Physical Layer Model}

The most commonly used radio model for the study of wireless networks is the socalled path loss model. This model assumes that the received signal power at distance $d$ is equal to $c \cdot d^{-\beta}$, where $c$ is a constant and $\beta$ is the path loss exponent. The path loss exponent depends on the environment and terrain structure and can vary between 
2 in free space to 6 in heavily built urban areas [11]. Normally the signal power at distance $d$ predicted by the path loss model is called the area mean power. Path loss model assumes radio signals can be received correctly when their power exceeds a minimum threshold value $\gamma$. With this assumption, the path loss model results into a perfect circular coverage area around each node with radius $\mathrm{R}=(c / \gamma) 1 / \beta$. However, this is an unrealistic assumption in most practical situations. In reality the received power levels may show significant variations around the area mean power [12].

The log-normal shadowing [13] model that we use in this paper is more realistic than the path loss model because it allows for random signal power variations. Due to those variations, the coverage area will deviate from a perfect circular shape and consequently, some short links could disappear while long links could emerge (see Figure 2).
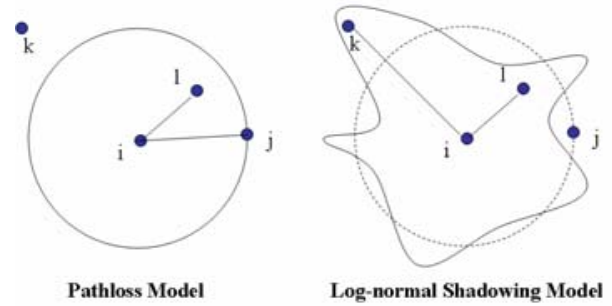

Fig.2. Coverage area comparison of Radio Models

The shadowing model consists of two parts. The first one is known as path loss model which predicts the mean received power at distance $d$, denoted by $\overline{P_{r}(d)}$. It uses a close-in distance $d_{0}$ as a reference. $\overline{P_{r}(d)}$ is computed relative to $P_{r}\left(d_{0}\right)$ as follows.

$$
\frac{P_{r}\left(d_{0}\right)}{\overline{P_{r}(d)}}=\left(\frac{d}{d_{0}}\right)^{\beta}
$$

$\beta$ is the path loss exponent and $P_{r}\left(d_{0}\right)$ can be computed from free space model. The path loss is usually measured in $\mathrm{dB}$. So from Eq. (1) we have

$$
\left[\overline{\frac{P_{r}(d)}{P_{r}\left(d_{0}\right)}}\right]_{d B}=-10 \beta \log \left(\frac{d}{d_{0}}\right) \text {. }
$$

The second part of the shadowing model reflects the variation of the received power at certain distance. It is a log-normal random variable, that is, it is of Gaussian distribution if measured in $\mathrm{dB}$. The overall shadowing model is represented by

$$
\left[\frac{P_{r}(d)}{P_{r}\left(d_{0}\right)}\right]_{d B}=-10 \beta \log \left(\frac{d}{d_{0}}\right)+X_{d B},
$$

where $X_{d B}$ is a Gaussian random variable with zero mean and standard deviation $\sigma_{d B}$. $\sigma_{d B}$ is called the shadowing deviation, and is also obtained by measurement. Eq. (3) is also known as a log-normal shadowing model.

\subsection{Antenna Model}


We study networks with not only omni antennas but also directional antennas. The use of directional antennas can permit energy savings and reduce interference by concentrating transmission energy where it is needed. We use a directional antenna propagation model [14] as shown in Fig. 3, where the antenna orientation $\varphi(0 \leqslant \varphi$ $<2 \pi$ ) of node is defined as the angle measured counter-clockwise from the horizontal axis to the antenna boresight, and the antenna directionality is specified as the angle of beamwidth $\theta_{f}\left(0 \leqslant \theta_{f}<2 \pi\right)$. Table 1 shows the antenna classification based on above model.

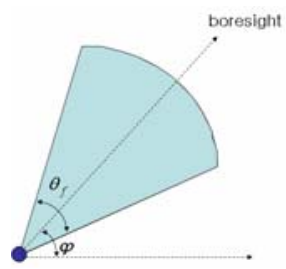

Fig.3. Directional antenna propagation model

Table 1: Antenna classification

\begin{tabular}{llll}
\hline & Omni-directional & $\begin{array}{c}\text { Modestly } \\
\text { directional }\end{array}$ & \multicolumn{1}{c}{$\begin{array}{c}\text { Highly } \\
\text { directional }\end{array}$} \\
\hline $\begin{array}{l}\text { Antenna Directionality } \\
\text { Antenna Orientation }\end{array}$ & fixed beamwidth & fixed beamwidth & $\begin{array}{l}\text { variable beamwidth } \\
\text { steerable }\end{array}$ \\
\hline
\end{tabular}

In this paper we only focus on the modestly directional antenna which has the following characteristics:

1. Beamwidth of each antenna cannot be adjusted, i.e., $\theta_{f}$ is fixed for any node.

2. Orientation of each antenna can be shifted to any desired direction to provide connectivity to a subset of the nodes that are within communication range.

3. A single antenna beam is provided for each session in which a node participates.

The transmission power needed by node $v$ to transmit to node $u$ in its antenna beam using beamwidth $\theta_{f}$ is

$$
c \cdot r^{\beta} \frac{\theta_{f}}{2 \pi}
$$

where $\beta$ is the path loss, $r$ is the transmission radius and $c$ is constant coefficient.

\subsection{Link Layer Model}

We consider two different operating models [15]:

a) End-to-End Retransmissions (EER): where the individual links do not provide link-layer retransmissions and error recovery.

b) Hop-by-Hop Retransmissions (HHR): where each individual link provides reliable forwarding to the next hop using localized packet retransmissions.

For HHR case, we employ a communication protocol between two nodes proposed in [8-10]. After receiving any packet from sender, the receiver sends $u$ acknowledge- 
ments. If the sender does not receive any acknowledgement, it will retransmit the packet. They also derive the expected number of messages in this protocol as measure of hop count between two nodes. The count includes transmissions by sender and acknowledgments by receiver. They assume both the acknowledgement and data packets are of the same length.

Let $S$ and $A$ be the sender and receiver nodes respectively, and let $|S A|=d$ be the distance between them. Probability that $A$ receives the packet from $S$ is $p(d)$. Probability that $S$ receives one particular packet from $A$ is $p(d)$ and the probability that it does not receive the packet is $1-p(d)$. Therefore, the probability that $S$ does not receive any of the $u$ acknowledgements is $(1-p(d))^{u}$. Thus, the probability that $S$ receives at least one of $u$ acknowledgements from $A$ is $1-(1-p(d))^{u}$. Therefore, $p(d)\left(1-(1-p(d))^{u}\right)$ is the probability that $S$ receives acknowledgement after sending a packet and therefore stops transmitting further packets. Thus, the expected number of packets at $S$ is $1 /\left[p(d)\left(1-(1-p(d))^{u}\right)\right]$. Each of these packets is received at $A$ with probability $p(d)$. If received correctly, it generates $u$ acknowledgements. The total expected number of acknowledgements sent by $A$ is then $u p(d) /\left[p(d)\left(1-(1-p(d))^{u}\right)\right]=u /\left[\left(1-(1-p(d))^{u}\right)\right]$. The total expected hop count between two nodes at distance $d$ is then $1 /\left[p(d)\left(1-(1-p(d))^{u}\right)\right]+u /\left[\left(1-(1-p(d))^{u}\right)\right]$.

\section{Metrics under Realistic Physical Layer Model}

The broken link problem caused by realistic physical layer is relatively easy to understand as shown in Fig. 2. Since energy efficient broadcast protocols aim to minimize energy consumption, they select a small forward node set and choose right the distance between senders and relay nodes as actual transmission radius. However, under realistic physical layer, e.g. shadowing model, they may get very poor network coverage because of link availability problem. In Fig. 4, node $j$ and $c$ have been selected as forward nodes, while because the broken link between source node $i$ and $j$, node $c, a$ and $b$ also can't be reached.

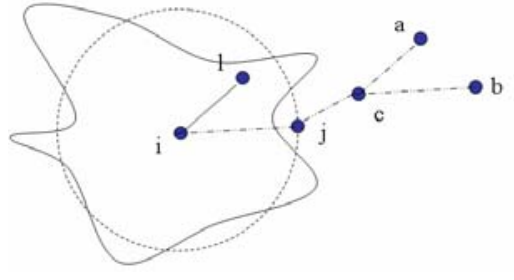

Fig. 4 Network coverage problem under practical model

In order to apply existing protocols without having to redesign them, the actual transmission radius for source node and forward nodes should be redesigned. To define appropriate transmission radius under practical models, we observed two different metrics: reception probability and expected energy consumption.

\subsection{Packet Reception Probability}


In shadowing model, the probability that the received power at a location $d$ exceeds a threshold value $\gamma$ can be given as:

$$
P_{r}\left[P_{r}(d)>\gamma\right]=0.5\left(1-\operatorname{erf}\left[\frac{\gamma-\overline{P_{r}(d)}}{\sqrt{2} \sigma}\right]\right)
$$

While exact computation of packet reception probability $p(d)$, for use in routing and broadcasting decision, is a time consuming process, and is based on several measurements (e.g. signal strengths, time delays and GPS) which cause some errors. It is therefore desirable to consider a reasonably accurate approximation that will be fast for use. I. Stojmenovic, et al [8-10] derives the approximation for probability of receiving a packet successfully as a function of distance $d$ between two nodes. Having in mind an error within $4 \%$ the model is

$$
p(r, d)= \begin{cases}1-\frac{\left(\frac{d}{r}\right)^{2 \beta}}{2} & 0 \leq d<r \\ \frac{\left(\frac{2 r-d}{r}\right)^{2} \beta}{2} & r \leq d \leq 2 r \\ 0 & \text { others }\end{cases}
$$

where $\beta$ is the power attenuation factor with fixed value between 2 and 6 , and $r$ is transmission radius with $p(r, d=r)=0.5$. Fig. 5 shows the packet reception probability with approximation $p(r, d)$ when $\beta$ is 2 .

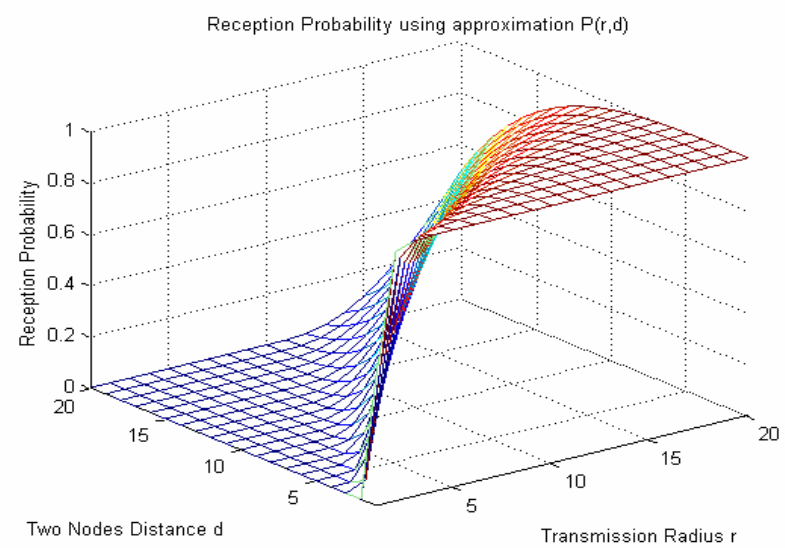

Fig. 5 Reception probability with approximation $p(r, d)$

\subsection{Expected Energy Consumption}

Assume now that two nodes are at distance $d$, but a packet is sent with transmission radius $r$ and with omni-antenna or fixed directional antenna $\theta_{f}$. The exact transmission power with omni-antenna is then $c r^{\beta}$ where $c$ is a constant; the exact transmission power with fixed directional-antenna $\theta_{f}$ is then ${ }_{c r} r^{\beta} \frac{\theta_{f}}{2 \pi}$. In addition, $c$ is assumed to be 1 for simplicity of analysis in next section. The packet reception probability at distance $d$ is $p(r, d)$. 
In EER framework, the sender sends a packet and the receiver may or may not receive the packet, which depends on the probability of receiving. Therefore, the expected energy consumption is $r^{\beta} \cdot p(r, d)$ for transmission between two nodes in net-

works with omni-antenna; $r^{\beta} \frac{\theta_{f}}{2 \pi} \cdot p(r, d)$ for transmission between two nodes with directional antenna of fixed beam width of $\theta_{f}$.

In HHR case, a message is retransmitted between two nodes until it is received and acknowledged correctly; after receiving any packet from the sender, the receiver sends acknowledgement. According to section 3.3 when $u$ equals 1 , the expected number of transmitted packets is $1 / p^{2}(r, d)$ and the expected number of acknowledgements is $1 / p(r, d)$. Therefore, the expected energy consumption is $r^{\beta}\left(1 / p^{2}(r, d)+1 / p(r, d)\right)$ for transmission between two nodes in networks with omni-antenna; $r^{\beta} \frac{\theta_{f}}{2 \pi}\left(1 / p^{2}(r, d)+1 / p(r, d)\right)$ for transmission between two nodes with directional antenna of fixed beam width of $\theta_{f}$. In addition, $\theta_{f}$ is assumed to be 1 for simplicity of energy analysis in next section.

\section{Analysis of Realistic Physical Layer Effect}

We extend broadcast oriented protocol to work in realistic physical layer environment: 1. Apply the selected broadcast oriented protocol to set up broadcast tree and determine the set of rely nodes.

2. Use $r$ for each rely node in the actual transmission.

Step 1 of the above process varies from protocol to protocol. As for the metric to decide the value of $r$, there exists a trade-off or negotiation between maximizing probability of delivery and minimizing energy consumption. We propose the following rules: for broadcasting in wireless network with omni-antennas, minimizing energy consumption is the primary metric; otherwise, for network with directional antennas, maximizing probability of delivery will be the primary metric, since transmission coverage overlapping is much fewer than that in networks with omni-antennas.

\subsection{EER Case}

In EER case, a sender sends a packet and a receiver may or may not receive the packet which depends on the reception probability. The reception probability function is $P(r, d)=\left(1-(d / r)^{2 \beta} / 2\right)$ for $d<r,((2 r-d) / r)^{2 \beta} / 2$ for $r \leq d \leq 2 r$, and 0 for all the other $d$ where $d$ is the distance before sender and receiver and $r$ is actual transmission range.

For network with directional antennas, since maximizing probability of delivery is our primary metric, at least we have to guarantee the reception probability no less than 0.5 ; however if the reception probability is near 1 , the energy consumption will be too high. Since after probability reaches around 0.9 , the acceleration of $P(r, d)$ curve decreases greatly, therefore we choose [0.5 0.9$]$ as the acceptable reception probability scope. From formula in section 4.1 we can find that if $r>d$, the scope of reception probability is $[0.5,1]$; otherwise, if $r<d$, reception probability will be less 
than 0.5 . Since we should guarantee the reception probability no less than 0.5 , we will only use $P(r, d)=\left(1-(d / r)^{2 \beta} / 2\right)$ for $d<r$. For any value of $\beta, 2 \leq \beta \leq 6$, if we want to get the relationship of $d$ and $r(r>d)$ for certain reception probability $a$, we can set up the formula as $1-(d / r)^{2 \beta} / 2=\alpha$, then we get $r=[2(1-\alpha)]^{-1 / 2 \beta} d$. Therefore, in order for reception probability to be in the range of [0.5 0.9], the transmission radius should be in the range of $\left[d(1 / 5)^{-1 / 2 \beta} d\right]$. We can verify it through Fig. 6 , where $\beta=2, d=10,20$ and 30. According to our proposal, we can choose the transmission radius in the scope of [10 15], [20 30] and [30 45] respectively. In Fig. 6(a), the according reception probability is in the scope of [0.5 0.9]; in Fig. 6(b), the according expected energy consumption is in the scope of [47 202], [197 811] and [447 1824] respectively.

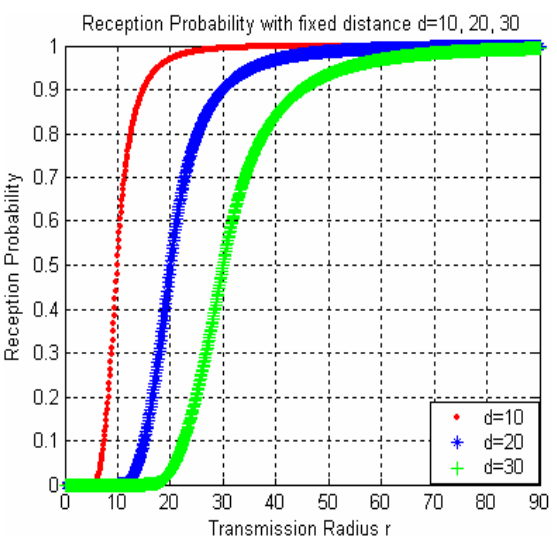

(a) Reception probability

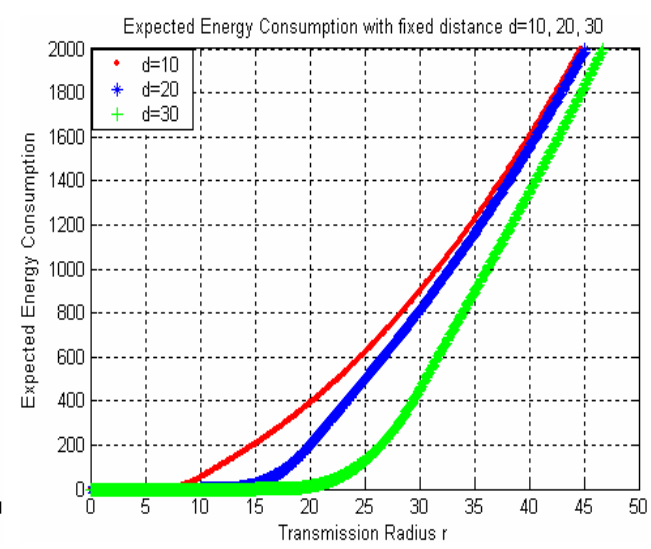

(b) Expected energy consumption

Fig. 6. Reception probability and expected energy consumption with fixed distance $d$

For network with omni-antennas, minimizing expected energy consumption is primary metric. We know as transmission $r$ increases, the expected energy consumption will also increase. Therefore, we want to choose the transmission radius $r$ value as small as possible. Whereas, even minimizing energy consumption is the primary metric, we still cannot neglect the reception probability. According our proposal above, which is selecting $r$ in the scope $\left[d(1 / 5)^{-1 / 2 \beta} d\right.$, and getting the reception probability scope [0.5 0.9$]$, by guaranteeing reception probability not less than $50 \%$, we decide to choose $d$ as the transmission radius $r$.

\subsection{HHR Case}

In HHR case, a message is retransmitted between two nodes until it is received and acknowledged correctly; after receiving any packet from sender, the receiver sends $u$ acknowledgements. Considering the characteristic of link layer in HHR case, it's better to be employed in networks with directional antennas, which represent one to one transmission model. In addition, we can find the link layer has already guaranteed successful reception, therefore our research moves to minimizing the expected energy consumption between two nodes. According to section 4.2, the total expected energy consumption is $r^{\beta}\left(1 / p^{2}(r, d)+1 / p(r, d)\right)$, that is, the combination of consumption at sender 
$S$ and receiver $A$. Therefore our work is transferred to maximize the reception probability at sender $S$ and receiver $A$.

For any value of $\beta, 2 \leq \beta \leq 6$, for receiver $A$, the relationship of $d$ and $r(r>d)$ for certain reception probability $a$ is $r=[2(1-\alpha)]^{-1 / 2 \beta} d$, then in order for reception probability to be in the range of [0.5 0.9], the transmission radius should be in the range of $\left[d(1 / 5)^{-1 / 2 \beta} d\right]$; however, for sender $S$, the relationship of $d$ and $r(r>d)$ for certain reception probability $a$ is $r=\left[2\left(1-\alpha^{1 / 2}\right)\right]^{-1 / 2 \beta} d$, then in order for reception probability

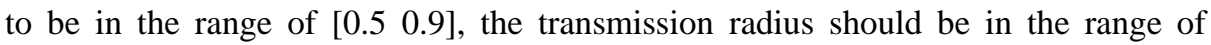
$\left[\left[2\left(1-(0.5)^{1 / 2}\right)\right]^{-1 / 2 \beta} d\left[2\left(1-(0.9)^{1 / 2}\right)\right]^{-1 / 2 \beta} d\right]$. Therefore considering the reception probability of both sender $S$ and receiver $A$, our proposal can be extended as the following: in HHR case, we choose $r$ from the scope of $\left[\left[2\left(1-(0.5)^{1 / 2}\right)\right]^{-1 / 2 \beta} d(1 / 5)^{-1 / 2 \beta} d\right]$, where for sender $S$ the scope of reception probability is $[0.50 .9)$ and for receiver $A$ the scope of reception probability is within $(0.50 .9]$. We can verify it through Fig. 7 , where $\beta=2, d=10,20$ and 30 . The reception probability at sender $S$ and at receiver $A$ with fixed distance $d$ when $\beta$ is 2 is showed in Fig. 7. According to our proposal, we can choose the transmission radius in the scope of [11.4, 15], [22.9 30] and [34.3 45] respectively. In Fig. 7, for sender $S$, the scope of reception probability is [0.5 0.8$]$ and for receiver $A$, the scope of reception probability is within [0.7 0.9].

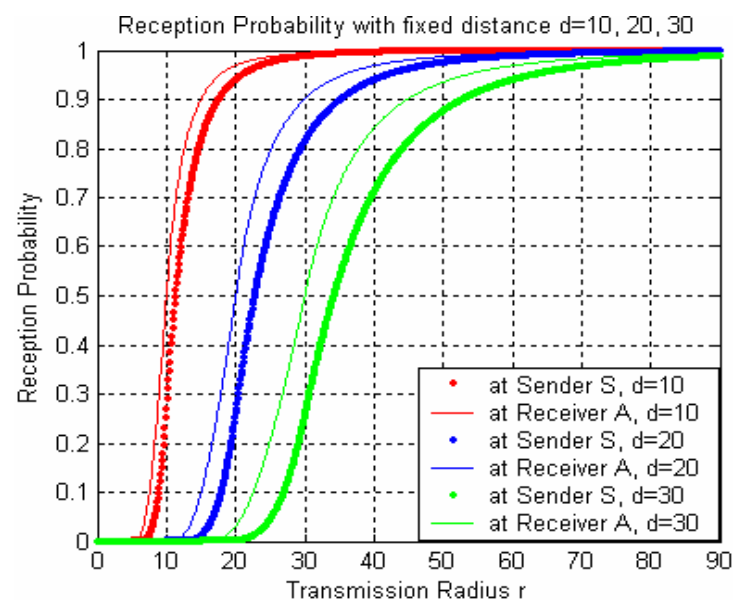

Fig. 7. Reception probability with fixed distance $d=10,20,30$

In HHR case, because of the characteristic of link layer, the number of transmission between two nodes is more than one, therefore expected hop count and expected energy consumption will be higher than that in EER case. Fig. 8 shows the total expected hop count and energy consumption including sender $S$ and receiver $A(\beta=2)$.

We can verify whether our proposal of choosing $r$ from the scope of $\left[\left[2\left(1-(0.5)^{1 / 2}\right)\right]^{-1 / 2 \beta} d(1 / 5)^{-1 / 2 \beta} d\right]$ is reasonable or not. The total expected hop count and energy consumption with fixed distance $d=10,20,30$ when $\beta$ is 2 is showed in Figure 8. According to our proposal, we can choose the transmission radius in the scope of [11.4 15], [22.9 30] and [34.3 45] respectively. Fig. 8(a) shows that if the 
transmission radius $r$ is not less than the distance 10, 20 and 30 respectively, expected hop count will be less than 5 and also at last decrease to a constant number. Fig. 8(b) shows that the expected energy consumption can get minimum value when $r$ is around 11.4, 22.9 and 34.3 respectively; whereas if $r$ is larger than those values, the expected energy consumption will increase. Therefore, even if $r$ is larger than 15, 30 and 45 respectively, we can get the minimum expected hop count, but because the expected energy consumption will be larger, so we still cannot choose $r$ larger than 15, 30 and 45 respectively. In a word, our proposal for HHR case is to choose the transmission radius $r$ in the scope of $\left[\left[2\left(1-(0.5)^{1 / 2}\right)\right]^{-1 / 2 \beta} d(1 / 5)^{-1 / 2 \beta} d\right]$, which can get good performance at expected hop count and energy consumption.

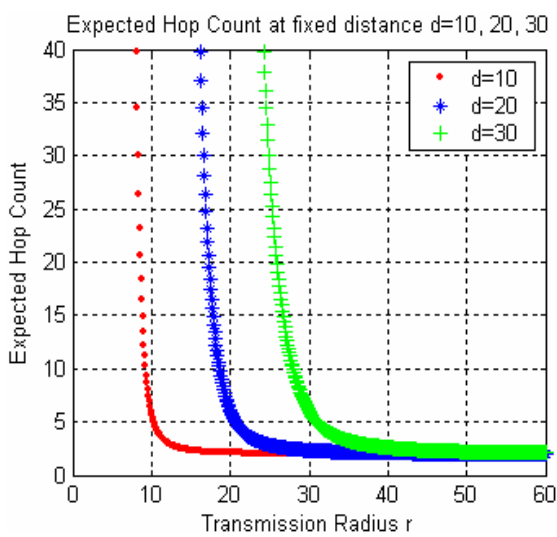

(a) Total expected hop count

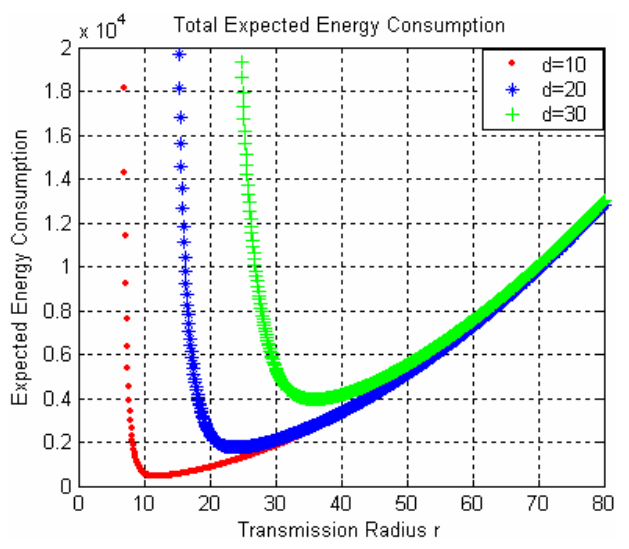

(b) Total expected energy consumption

Fig. 8. Total expected hop count and energy consumption with fixed distance $d$

\section{Conclusions}

In this paper we investigated energy efficient broadcast protocols with and without acknowledgements and presented the trade off between maximizing probability of delivery and minimizing energy consumption for ad hoc wireless networks with realistic physical layer. In EER case, for network with omni-antennas, we decide to choose the distance $d$ between two nodes as transmission radius; in network with directional antenna, we propose to choose the transmission radius in the scope of $[d$ $\left.(1 / 5)^{-1 / 2 \beta} d\right]$ to maximize the probability of delivery. In HHR case, the link layer protocol is not suitable to one-to-all communication; therefore we only consider networks with directional antennas. We propose to choose the transmission radius in the scope of $\left[\left[2\left(1-(0.5)^{1 / 2}\right)\right]^{-1 / 2 \beta} d(1 / 5)^{-1 / 2 \beta} d\right]$, which can get good performance at expected hop count and energy consumption.

Currently, we are designing new broadcast protocols based on our analysis and we will compare the performance of our new protocols with that of existing broadcast protocols under realistic physical layer. 


\section{Acknowledgement}

The research was supported by the Driving Force Project for the Next Generation of Gyeonggi Provincial Government in Republic of Korea.

\section{References}

1. S. Basagni, M. Conti, S. Giordano, I. Stojmenovic (eds.): Mobile Ad Hoc Networking. IEEE Press/Wiley, July (2004)

2. J. M. Kahn, R. H. Katz, and K. S. J. Pister: Next century challenges: Mobile networking for "smart dust". International Conference on Mobile Computing and Networking (MOBICOM) (1999) 271-278

3. G. Giordano, I. Stojmenovic (ed.): Mobile Ad Hoc Networks. Handbook of Wireless Networks and Mobile Computing, Wiley (2002) 325-346

4. G.J. Pottie and W.J. Kaiser: Wireless integrated network sensors. Communications of the ACM, vol. 43, no. 5 (2000) 551-558

5. J. E. Wieselthier, G. D. Nguyen, and A. Ephremides: On the construction of energy-efficient broadcast and multicast trees in wireless networks. Proc. IEEE INFOCOM, Mar (2000) 585594

6. J.E. Wieselthier, G.D. Nguyen, A. Ephremides: Energy-Limited Wireless Networking with Directional Antennas: The Case of Session-Based Multicasting. Proc. IEEE INFOCOM (2002) 190-199

7. Mineo Takai, Jay Martin and Rajive Bagrodia: Effects of wireless physical layer modeling in mobile ad hoc networks. Proceedings of the 2nd ACM international symposium on Mobile ad hoc networking \& computing (2001) 87-94

8. I. Stojmenovic, A. Nayak, J. Kuruvila, F. Ovalle-Martinez and E. Villanueva-Pena: Physical layer impact on the design of routing and broadcasting protocols in ad hoc and sensor networks. Journal of Computer Communications (Elsevier), Special issue on Performance Issues of Wireless LANs, PANs, and Ad Hoc Networks, Dec (2004)

9. Johnson Kuruvila, Amiya Nayak and Ivan Stojmenovic: Hop count optimal position based packet routing algorithms for ad hoc wireless networks with a realistic physical layer. IEEE Journal on selected areas in communications, vol.23, no.6, June (2005)

10. J. Kuruvila, A. Nayak, I. Stojmenovic: Greedy localized routing for maximizing probability of delivery in wireless ad hoc networks with a realistic physical layer. Algorithms for Wireless And mobile Networks (A_SWAN) Personal, Sensor, Ad-hoc, Cellular Workshop, at Mobiquitous, Boston, August (2004) 22-26

11. T. Rappaport: Wireless Communications, Principles and Practice. Upper Saddle River Prentice-Hall PTR (2002)

12. R. Hekmat, Piet Van Mieghem: Interference Power Sum with Log-Normal Components in Ad-Hoc and Sensor Networks. WiOpt (2005) 174-182

13. Network Simulator - ns-2, http://www.isi.edu/nsnam/ns/.

14. Song Guo, Oliver W. W. Yang: Antenna orientation optimization for minimum-energy multicast tree construction in wireless ad hoc networks with directional antennas. MobiHoc (2004) 234-243

15. Suman Banerjee, Archan Misra: Minimum energy paths for reliable communication in multi-hop wireless networks. MobiHoc, Switzerland (2002) 146-156 